\title{
A family of refinements of Heinz inequalities of matrices
}

\author{
Hassane Abbas* and Bassam Mourad
}

"Correspondence: habbas@ul.edu.lb Department of Mathematics,

Faculty of Sciences, Lebanese University, Hadath, Beirut, Lebanon

\begin{abstract}
For any unitarily invariant norm \|\|\|\| , the Heinz inequalities for operators assert that $2\left\|A^{\frac{1}{2}} X B^{\frac{1}{2}}\right\| \leq\left\|A^{v} X B^{1-v}+A^{1-v} X B^{v}\right\| \leq\|\| A X+X B \|$, for $A, B$, and $X$ any operators on a complex separable Hilbert space such that $A, B$ are positive and $v \in[0,1]$. In this paper, we obtain a family of refinements of these norm inequalities by using the convexity of the function $f(v)=\left\|A^{v} X B^{1-v}+A^{1-v} X B^{v}\right\|$ and the Hermite-Hadamard inequality.
\end{abstract}

Keywords: Heinz inequality; convex function; Hermite-Hadamard inequality; unitarily invariant norm

\section{Introduction}

Let $M_{n}(\mathbf{C})$ be the algebra of $n \times n$ complex matrices. We denote by $H_{n}(\mathbf{C})$ the set of all Hermitian matrices in $M_{n}(\mathbf{C})$. The set of all positive semi-definite matrices in $M_{n}(\mathbf{C})$ shall be denoted by $H_{n}^{+}(\mathbf{C})$. A norm $\|\cdot \mid\|$ on $M_{n}(\mathbf{C})$ is called unitarily invariant or symmetric if

$$
\|U A V\||=||A \||
$$

for all $A \in M_{n}(\mathbf{C})$ and for all unitaries $U, V \in M_{n}(\mathbf{C})$.

The arithmetic-geometric mean inequality for two nonnegative real numbers $a$ and $b$ is

$$
\sqrt{a b} \leq \frac{a+b}{2}
$$

which has been generalized to the context of matrices as follows:

$$
2\left\|A^{\frac{1}{2}} X B^{\frac{1}{2}}\right\| \leq\|\| A X+X B \|,
$$

where $A, B \in H_{n}^{+}(\mathbf{C}), X \in M_{n}$, and $\|\cdot \cdot\|$ is a unitarily invariant norm on $M_{n}(\mathbf{C})$.

For $v \in[0,1]$ and two nonnegative numbers $a$ and $b$, the Heinz mean is defined as

$$
H_{v}(a, b)=\frac{a^{v} b^{1-v}+a^{1-v} b^{v}}{2} .
$$

Clearly the Heinz mean interpolates between the geometric mean and the arithmetic mean:

$$
\sqrt{a b} \leq H_{v}(a, b) \leq \frac{a+b}{2} .
$$

O2014 Abbas and Mourad; licensee Springer. This is an Open Access article distributed under the terms of the Creative Commons Attribution License (http://creativecommons.org/licenses/by/2.0), which permits unrestricted use, distribution, and reproduction in any medium, provided the original work is properly cited. 
The function $H_{v}(a, b)$ has the following properties: it is convex, attains its minimum at $v=\frac{1}{2}$, its maximum at $v=0$ and $v=1$, and $H_{v}(a, b)=H_{1-v}(a, b)$ for $0 \leq v \leq 1$. The generalization of the above inequalities to matrices is due to Bhatia and Davis [1] as follows:

$$
2\left\|A^{\frac{1}{2}} X B^{\frac{1}{2}}\right\| \leq\left\|A^{v} X B^{1-v}+A^{1-v} X B^{v}\right\| \leq\|A X+X B\|,
$$

where $A, B \in H_{n}^{+}(\mathbf{C}), X \in M_{n}(\mathbf{C})$, and $v \in[0,1]$. For a historical background and proofs of these norm inequalities as well as their refinements, and diverse applications, we refer the reader to the [2-8], and the references therein. Indeed, it has been proved, in [1], that $f(v)=\left\|A^{v} X B^{1-v}+A^{1-v} X B^{v}\right\|$ is a convex function of $v$ on $[0,1]$ with symmetry about $v=\frac{1}{2}$, and attains its minimum there and it has a maximum at $v=0$ and $v=1$. Moreover, it increases on $\left[0, \frac{1}{2}\right]$ and decreases on $\left[\frac{1}{2}, 1\right]$.

In $[4,5],(1.1)$ is refined by using the so-called Hermite-Hadamard inequality:

$$
g\left(\frac{a+b}{2}\right) \leq \frac{1}{b-a} \int_{a}^{b} g(t) d t \leq \frac{g(a)+g(b)}{2},
$$

where $g$ is a convex function on $[a, b]$.

Recently, in [3] and [7], respectively, the following inequalities were used to get new refinements of (1.1):

$$
\begin{aligned}
& g\left(\frac{a+b}{2}\right) \leq \frac{1}{b-a} \int_{a}^{b} g(t) d t \leq \frac{1}{4}\left(g(a)+2 g\left(\frac{a+b}{2}\right)+g(b)\right) \leq \frac{g(a)+g(b)}{2}, \\
& g\left(\frac{a+b}{2}\right) \leq \frac{1}{b-a} \int_{a}^{b} g(t) d t \leq \frac{1}{32}\left(15 g(a)+2 g\left(\frac{a+b}{2}\right)+15 g(b)\right) \leq \frac{g(a)+g(b)}{2} .
\end{aligned}
$$

The purpose of this note is to obtain a family of new refinements of Heinz inequalities for matrices. Also the two refinements, given in [3] and [7], are two special cases of this new family.

\section{Main results}

We start by the following key lemma which plays a central role in our investigation to obtain a further series of refinements of the Heinz inequalities.

Lemma 1 Let $g$ be a convex function on the interval $[a, b]$. Then for any positive integer $n$, we have

$$
\begin{aligned}
g\left(\frac{a+b}{2}\right) & \leq \frac{1}{b-a} \int_{a}^{b} g(t) d t \leq \frac{1}{4 n}\left[(2 n-1) g(a)+2 g\left(\frac{a+b}{2}\right)+(2 n-1) g(b)\right] \\
& \leq \frac{g(a)+g(b)}{2} .
\end{aligned}
$$

Proof Since $g$ is convex on $[a, b]$, we have

$$
g\left(\frac{a+b}{2}\right) \leq \frac{g(a)+g(b)}{2} .
$$


Thus

$$
(2 n-1) g(a)+2 g\left(\frac{a+b}{2}\right)+(2 n-1) g(b) \leq 2 n g(a)+2 n g(b),
$$

whence

$$
\frac{1}{4 n}\left((2 n-1) g(a)+2 g\left(\frac{a+b}{2}\right)+(2 n-1) g(b)\right) \leq \frac{g(a)+g(b)}{2} .
$$

To prove the middle inequality, we start by

$$
\begin{aligned}
\frac{1}{b-a} \int_{a}^{b} g(t) d t & =\frac{1}{b-a}\left[\int_{a}^{\frac{a+b}{2}} g(t) d t+\int_{\frac{a+b}{2}}^{b} g(t) d t\right] \\
& \leq \frac{1}{b-a}\left[\frac{g\left(\frac{a+b}{2}\right)+g(a)}{2} \cdot \frac{b-a}{2}+\frac{g(b)+g\left(\frac{a+b}{2}\right)}{2} \cdot \frac{b-a}{2}\right] \\
& =\frac{1}{4}\left[g(a)+2 g\left(\frac{a+b}{2}\right)+g(b)\right] \\
& =\frac{1}{4 n}\left[n g(a)+2 n g\left(\frac{a+b}{2}\right)+n g(b)\right] \\
& =\frac{1}{4 n}\left[n g(a)+2 g\left(\frac{a+b}{2}\right)+(2 n-2) g\left(\frac{a+b}{2}\right)+n g(b)\right] \\
& \leq \frac{1}{4 n}\left[n g(a)+2 g\left(\frac{a+b}{2}\right)+(2 n-2)\left[\frac{g(a)+g(b)}{2}\right]+n g(b)\right] \\
& =\frac{1}{4 n}\left[n g(a)+2 g\left(\frac{a+b}{2}\right)+(n-1) g(a)+(n-1) g(b)+n g(b)\right] \\
& =\frac{1}{4 n}\left[(2 n-1) g(a)+2 g\left(\frac{a+b}{2}\right)+(2 n-1) g(b)\right] .
\end{aligned}
$$

Applying the previous lemma on the convex function defined earlier

$$
f(v)=\left\|A^{v} X B^{1-v}+A^{1-v} X B^{v}\right\|
$$

on the interval $[\mu, 1-\mu]$ when $0 \leq \mu \leq \frac{1}{2}$ and on the interval $[1-\mu, \mu]$ when $\frac{1}{2} \leq \mu \leq 1$, we obtain the following refinement of the first inequality (1.1) which is a kind of refinements of Theorem 1 in a paper Kittaneh [5] and Theorem 1 in a paper of Feng [3].

Theorem 1 Let $A, B \in H_{n}^{+}(\mathbf{C})$, and $X \in M_{n}(\mathbf{C})$. Let $n$ be any positive integer. Then for any $\mu \in[0,1]$, and for every unitarily invariant norm $\|\cdot\| \mid \|$ on $M_{n}(\mathbf{C})$, we have

$$
\begin{aligned}
2\left\|A^{\frac{1}{2}} X B^{\frac{1}{2}}\right\| & \leq \frac{1}{|1-2 \mu|}\left|\int_{\mu}^{1-\mu}\left\|A^{v} X B^{1-\nu}+A^{1-\nu} X B^{v}\right\| d \nu\right| \\
& \leq \frac{1}{2 n}\left[(2 n-1)\left\|A^{\mu} X B^{1-\mu}+A^{1-\mu} X B^{\mu}\right\|+2\left\|A^{\frac{1}{2}} X B^{\frac{1}{2}}\right\|\right] \\
& \leq\left\|A^{\mu} X B^{1-\mu}+A^{1-\mu} X B^{\mu}\right\| .
\end{aligned}
$$


Proof First assume that $0 \leq \mu \leq \frac{1}{2}$. Then it follows from Lemma 1 that

$$
\begin{aligned}
f\left(\frac{\mu+1-\mu}{2}\right) & \leq \frac{1}{1-2 \mu} \int_{\mu}^{1-\mu} f(t) d t \\
& \leq \frac{1}{4 n}\left[(2 n-1) f(\mu)+2 f\left(\frac{1-\mu+\mu}{2}\right)+(2 n-1) f(1-\mu)\right] \\
& \leq \frac{f(\mu)+f(1-\mu)}{2}=f(\mu) .
\end{aligned}
$$

Since $f(\mu)=f(1-\mu)$, we have

$$
\begin{aligned}
f\left(\frac{1}{2}\right) & \leq \frac{1}{1-2 \mu} \int_{\mu}^{1-\mu} f(t) d t \leq \frac{1}{4 n}\left[(4 n-2) f(\mu)+2 f\left(\frac{1}{2}\right)\right] \\
& \leq \frac{1}{2 n}\left[(2 n-1) f(\mu)+f\left(\frac{1}{2}\right)\right] \leq f(\mu) .
\end{aligned}
$$

Thus,

$$
\begin{aligned}
2\left\|A^{\frac{1}{2}} X B^{\frac{1}{2}}\right\| & \leq \frac{1}{1-2 \mu} \int_{\mu}^{1-\mu}\left\|A^{v} X B^{1-\nu}+A^{1-v} X B^{v}\right\| d \nu \\
& \leq \frac{1}{2 n}\left[(2 n-1)\left\|A^{\mu} X B^{1-\mu}+A^{1-\mu} X B^{\mu}\right\|+2\left\|A^{\frac{1}{2}} X B^{\frac{1}{2}}\right\|\right] \\
& \leq\left\|A^{\mu} X B^{1-\mu}+A^{1-\mu} X B^{\mu}\right\| .
\end{aligned}
$$

Now, assume that $\frac{1}{2} \leq \mu \leq 1$. Then, by applying (2.2) to $1-\mu$, it follows that

$$
\begin{aligned}
2\left\|A^{\frac{1}{2}} X B^{\frac{1}{2}}\right\| & \leq \frac{1}{2 \mu-1} \int_{1-\mu}^{\mu}\left\|A^{\nu} X B^{1-\nu}+A^{1-\nu} X B^{\nu}\right\| d v \\
& \leq \frac{1}{2 n}\left[(2 n-1)\left\|A^{\mu} X B^{1-\mu}+A^{1-\mu} X B^{\mu}\right\|+2\left\|A^{\frac{1}{2}} X B^{\frac{1}{2}}\right\|\right] \\
& \leq\left\|A^{\mu} X B^{1-\mu}+A^{1-\mu} X B^{\mu}\right\| .
\end{aligned}
$$

Since

$$
\begin{aligned}
& \lim _{\mu \rightarrow \frac{1}{2}} \frac{1}{|1-2 \mu|}\left|\int_{\mu}^{1-\mu}\left\|A^{v} X B^{1-v}+A^{1-v} X B^{v}\right\| d v\right| \\
& =\lim _{\mu \rightarrow \frac{1}{2}} \frac{1}{4 n}\left[(4 n-2) f(\mu)+f\left(\frac{1}{2}\right)\right]=2\left\|A^{\frac{1}{2}} X B^{\frac{1}{2}}\right\|,
\end{aligned}
$$

the inequalities in (2.1) follow by combining (2.2) and (2.3) and so the required result is proved.

Applying Lemma 1 to the function $f(v)=\left\|A^{v} X B^{1-v}+A^{1-v} X B^{v}\right\|$ in the interval $\left[\mu, \frac{1}{2}\right]$ on $0 \leq \mu \leq \frac{1}{2}$, and in the interval $\left[\frac{1}{2}, \mu\right]$ for $\frac{1}{2} \leq \mu \leq 1$, we obtain the following, which is a kind of refinements of Theorem 2 in a paper Kittaneh [5] and Theorem 2 in a paper of Feng [3]. 
Theorem 2 Let $A, B \in H_{n}^{+}(\mathbf{C})$, and $X \in M_{n}(\mathbf{C})$. Then, for any positive integer $n$, any $\mu \in$ $[0,1]$, and for every unitarily invariant norm $\|\cdot \cdot\|$ on $M_{n}(\mathbf{C})$, we have

$$
\begin{aligned}
&\left\|A^{\frac{1+2 \mu}{4}} X B^{\frac{3-2 \mu}{4}}+A^{\frac{3-2 \mu}{4}} X B^{\frac{1+2 \mu}{4}}\right\| \\
& \leq \frac{2}{|1-2 \mu|}\left|\int_{\mu}^{\frac{1}{2}}\left\|A^{v} X B^{1-v}+A^{1-\nu} X B^{v}\right\| d \nu\right| \\
& \leq \frac{1}{4 n}\left[(2 n-1)\left\|A^{\mu} X B^{1-\mu}+A^{1-\mu} X B^{\mu}\right\|+2\left\|A^{\frac{1+2 \mu}{4}} X B^{\frac{3-2 \mu}{4}}+A^{\frac{3-2 \mu}{4}} X B^{\frac{1+2 \mu}{4}}\right\|\right. \\
&\left.+2(2 n-1)\left\|A^{\frac{1}{2}} X B^{\frac{1}{2}}\right\|\right] \\
& \leq \frac{1}{2}\left[\left\|A^{\mu} X B^{1-\mu}+A^{1-\mu} X B^{\mu}\right\|+2\left\|A^{\frac{1}{2}} X B^{\frac{1}{2}}\right\|\right] .
\end{aligned}
$$

Inequalities (2.4) and the first inequality in (1.1) yield the following refinements of the first inequality in (1.1).

Corollary 1 Let $A, B \in H_{n}^{+}(\mathbf{C})$, and $X \in M_{n}(\mathbf{C})$. Then, for any positive integer $n$, any $\mu \in$ $[0,1]$, and for every unitarily invariant norm $\|\mid \cdot\|$ on $X \in M_{n}(\mathbf{C})$, we have

$$
\begin{aligned}
2\left\|A^{\frac{1}{2}} X B^{\frac{1}{2}}\right\| \leq & \left\|A^{\frac{1+2 \mu}{4}} X B^{\frac{3-2 \mu}{4}}+A^{\frac{3-2 \mu}{4}} X B^{\frac{1+2 \mu}{4}}\right\| \\
\leq & \frac{2}{|1-2 \mu|}\left|\int_{\mu}^{\frac{1}{2}}\left\|A^{v} X B^{1-\nu}+A^{1-\nu} X B^{\nu}\right\| d \nu\right| \\
\leq & \frac{1}{4 n}\left[(2 n-1)\left\|A^{\mu} X B^{1-\mu}+A^{1-\mu} X B^{\mu}\right\|\right. \\
& \left.+2\left\|A^{\frac{1+2 \mu}{4}} X B^{\frac{3-2 \mu}{4}}+A^{\frac{3-2 \mu}{4}} X B^{\frac{1+2 \mu}{4}}\right\|+2(2 n-1)\left\|A^{\frac{1}{2}} X B^{\frac{1}{2}}\right\|\right] \\
\leq & \frac{1}{2}\left[\left\|A^{\mu} X B^{1-\mu}+A^{1-\mu} X B^{\mu}\right\|+2\left\|A^{\frac{1}{2}} X B^{\frac{1}{2}}\right\|\right] \\
\leq & \left\|A^{\mu} X B^{1-\mu}+A^{1-\mu} X B^{\mu}\right\| .
\end{aligned}
$$

Applying the Lemma 1 to the function $f(v)=\left\|A^{v} X B^{1-v}+A^{1-v} X B^{v}\right\|$ on the interval $\left[\mu, \frac{1}{2}\right]$ when $0 \leq \mu \leq \frac{1}{2}$, and on the interval $\left[\frac{1}{2}, \mu\right]$ when $\frac{1}{2} \leq \mu \leq 1$, we obtain the following theorem, which is a kind of refinements of Theorem 3 in a paper Kittaneh [5] and Theorem 3 in a paper of Feng [3].

Theorem 3 Let $A, B \in H_{n}^{+}(\mathbf{C})$, and $X \in M_{n}(\mathbf{C})$ and let $n$ be a positive integer. Then:

(1) for any $0 \leq \mu \leq \frac{1}{2}$ and for every unitarily invariant norm $\|\cdot\| \|$, we have

$$
\begin{aligned}
&\left\|A^{\frac{\mu}{2}} X B^{1-\frac{\mu}{2}}+A^{1-\frac{\mu}{2}} X B^{\frac{\mu}{2}}\right\| \\
& \leq \frac{1}{\mu} \int_{0}^{\mu}\left\|A^{v} X B^{1-\nu}+A^{1-\nu} X B^{v}\right\| d v \\
& \leq \frac{1}{4 n}\left[(2 n-1)\|A X+X B\|+2\left\|A^{\frac{\mu}{2}} X B^{1-\frac{\mu}{2}}+A^{1-\frac{\mu}{2}} X B^{\frac{\mu}{2}}\right\|\right. \\
&\left.+(2 n-1)\left\|A^{\mu} X B^{1-\mu}+A^{1-\mu} X B^{\mu}\right\|\right] \\
& \leq \frac{1}{2}\left[\|A X+X B\|+\left\|A^{\mu} X B^{1-\mu}+A^{1-\mu} X B^{\mu}\right\|\right] ;
\end{aligned}
$$


(2) for any $\frac{1}{2} \leq \mu \leq 1$ and for every unitarily invariant norm $\|\cdot\| \|$, we have

$$
\begin{aligned}
& \left\|A^{\frac{1+\mu}{2}} X B^{\frac{1-\mu}{2}}+A^{\frac{1-\mu}{2}} X B^{\frac{1+\mu}{2}}\right\| \\
& \quad \leq \frac{1}{1-\mu} \int_{\mu}^{1}\left\|A^{\nu} X B^{1-\nu}+A^{1-v} X B^{\nu}\right\| d v \\
& \quad \leq \frac{1}{4 n}\left[(2 n-1)\|A X+X B\|+2\left\|A^{\frac{1+\mu}{2}} X B^{\frac{1-\mu}{2}}+A^{\frac{1-\mu}{2}} X B^{\frac{1+\mu}{2}}\right\|\right] \\
& \quad \leq \frac{1}{2}\left[\|A X+X B\|+\left\|A^{\mu} X B^{1-\mu}+A^{1-\mu} X B^{\mu}\right\|\right] .
\end{aligned}
$$

Since the function $f(v)=\left\|A^{v} X B^{1-v}+A^{1-v} X B^{v}\right\|$ is decreasing on the interval $\left[0, \frac{1}{2}\right]$ and increasing on the interval $\left[\frac{1}{2}, 1\right]$, and using the inequalities (2.6) and (2.7), we obtain a family of refinements of second inequality in (1.1).

Corollary 2 Let $A, B \in H_{n}^{+}(\mathbf{C})$, and $X \in M_{n}(\mathbf{C})$ and let $n$ be a positive integer. Then:

(1) for any $0 \leq \mu \leq \frac{1}{2}$ and for every unitarily invariant norm $\|\cdot\| \|$, we have

$$
\begin{aligned}
&\left\|A^{\mu} X B^{1-\mu}+A^{1-\mu} X B^{\mu}\right\| \\
& \leq\left\|A^{\frac{\mu}{2}} X B^{1-\frac{\mu}{2}}+A^{1-\frac{\mu}{2}} X B^{\frac{\mu}{2}}\right\| \\
& \leq \frac{1}{\mu} \int_{0}^{\mu}\left\|A^{\nu} X B^{1-\nu}+A^{1-\nu} X B^{\nu}\right\| d \nu \\
& \leq \frac{1}{4 n}\left[(2 n-1)\|A X+X B\|+2\left\|A^{\frac{\mu}{2}} X B^{1-\frac{\mu}{2}}+A^{1-\frac{\mu}{2}} X B^{\frac{\mu}{2}}\right\|\right. \\
&\left.+(2 n-1)\left\|A^{\mu} X B^{1-\mu}+A^{1-\mu} X B^{\mu}\right\|\right] \\
& \leq \frac{1}{2}\left[\|A X+X B\|+\left\|A^{\mu} X B^{1-\mu}+A^{1-\mu} X B^{\mu}\right\|\right] \\
& \leq\|A X+X B\| ;
\end{aligned}
$$

(2) for any $\frac{1}{2} \leq \mu \leq 1$ and for every unitarily invariant norm $\|\cdot\| \|$, we have

$$
\begin{aligned}
&\left\|A^{\mu} X B^{1-\mu}+A^{1-\mu} X B^{\mu}\right\| \\
& \leq\left\|A^{\frac{1+\mu}{2}} X B^{\frac{1-\mu}{2}}+A^{\frac{1-\mu}{2}} X B^{\frac{1+\mu}{2}}\right\| \\
& \leq \frac{1}{1-\mu} \int_{\mu}^{1}\left\|A^{v} X B^{1-\nu}+A^{1-v} X B^{v}\right\| d v \\
& \leq \frac{1}{4 n}\left[(2 n-1)\|A X+X B\|+2\left\|A^{\frac{1+\mu}{2}} X B^{\frac{1-\mu}{2}}+A^{\frac{1-\mu}{2}} X B^{\frac{1+\mu}{2}}\right\|\right. \\
&\left.+(2 n-1)\left\|A^{\mu} X B^{1-\mu}+A^{1-\mu} X B^{\mu}\right\|\right] \\
& \leq \frac{1}{2}\left[\|A X+X B\|+\left\|A^{\mu} X B^{1-\mu}+A^{1-\mu} X B^{\mu}\right\|\right] \\
& \leq\|A X+X B\| .
\end{aligned}
$$


It should be noted that in inequalities (2.8) and (2.9), we have

$$
\begin{aligned}
& \lim _{\mu \rightarrow 0 \frac{1}{\mu}} \int_{0}^{\mu}\left\|A^{v} X B^{1-v}+A^{1-v} X B^{v}\right\| d v \\
& \quad=\lim _{\mu \rightarrow 1} \frac{1}{1-\mu} \int_{\mu}^{1}\left\|A^{v} X B^{1-v}+A^{1-v} X B^{v}\right\| d v=\|A X+X B\| .
\end{aligned}
$$

Remark 1 The two special values $n=1$ and $n=8$ give the refinements of Heinz inequalities obtained in [3] and [7], respectively.

\section{Competing interests}

The authors declare that they have no competing interests.

\section{Authors' contributions}

The authors contributed equally to the manuscript and read and approved the final manuscript.

\section{Acknowledgements}

Thanks for both reviewers for their helpful comments and suggestions. The authors wish also to express their thanks to professor Mohammad S Moslehian for helpful suggestions for revising the manuscript. This research is supported by the Lebanese University grants program for the Discrete Mathematics and Algebra group.

Received: 17 February 2014 Accepted: 30 June 2014 Published: 22 Jul 2014

\section{References}

1. Bhatia, R: Matrix Analysis. Springer, New York (1997)

2. Bhatia, R, Davis, C: More matrix forms of the arithmetic-geometric mean inequality. SIAM J. Matrix Anal. Appl. 14 132-136 (1993)

3. Feng, Y: Refinements of Heinz inequalities. J. Inequal. Appl. 2012, 18 (2012)

4. Kittaneh, F, Manasrah, Y: Improved Young and Heinz inequalities for matrices. J. Math. Anal. Appl. 361, 262-269 (2010)

5. Kittaneh, F: On the convexity of the Heinz mean. Integral Equ. Oper. Theory 68, 519-527 (2010)

6. Kaur, R, Moslehian, MS, Singh, M, Conde, C: Further refinements of the Heinz. J. Linear algebra and its applications (Available online 27 February 2013)

7. Wang, S: Some new refinements of Heinz inequalities for matrices. J. Inequal. Appl. 1, 132-136 (2013)

8. Zou, L, He, C: On some inequalities for unitarily invariant norms and singular values. Linear Algebra Appl. 436, 3354-3361 (2012)

10.1186/1029-242X-2014-267

Cite this article as: Abbas and Mourad: A family of refinements of Heinz inequalities of matrices. Journal of Inequalities and Applications 2014, 2014:267

\section{Submit your manuscript to a SpringerOpen ${ }^{\ominus}$ journal and benefit from:}

- Convenient online submission

Rigorous peer review

- Immediate publication on acceptance

- Open access: articles freely available online

- High visibility within the field

- Retaining the copyright to your article 